\title{
АСИМПТОТИКА РЕШЕНИЯ ЗАДАЧИ ОПТИМАЛЬНОГО ГРАНИЧНОГО УПРАВЛЕНИЯ С ДВУМЯ МАЛЫМИ СОПОДЧИНЕННЫМИ ПАРАМЕТРАМИ
}

\section{А. Р. Данилин}

Рассматривается задача оптимального граничного управления решениями уравнения эллиптического типа в ограниченной области с гладкой границей с малым коэффициентом при операторе Лапласа и малым, соподчиненным с первым, коэффициентом при граничном условии и интегральными ограничениями на управление.

$$
\begin{cases}\mathcal{L}_{\varepsilon}:=-\varepsilon^{2} \Delta z+a(x) z=f(x), & x \in \Omega, \quad z \in H^{1}(\Omega), \\ l_{\varepsilon, \beta} z:=\varepsilon^{\beta} \frac{\partial z}{\partial n}=g(x)+u(x), & x \in \Gamma,\end{cases}
$$

со следующим функционалом качества

$$
J(u):=\left\|z-z_{d}\right\|^{2}+\nu^{-1}\|\| u \|^{2} \rightarrow \inf , \quad u \in \mathcal{U}
$$

где $0<\varepsilon \ll 1, \beta \geqslant 0, \beta \in \mathbb{Q}, \nu>0, H^{1}(\Omega)$ - соболевское пространство функций, $\partial z / \partial n-$ производная функции $z$ в точке $x \in \Gamma$ по направлению внешней (по отношению к области $\Omega$ ) нормали,

$$
\begin{gathered}
a(\cdot), f(\cdot), z_{d}(\cdot) \in C^{\infty}(\bar{\Omega}), \quad g(\cdot) \in C^{\infty}(\Gamma), \quad \forall x \in \bar{\Omega} \quad a(x) \geqslant \alpha^{2}>0, \\
\mathcal{U}=\mathcal{U}_{1}, \quad \mathcal{U}_{r}:=\left\{u(\cdot) \in L_{2}(\Gamma):\||| u \mid\| \leqslant r\right\} .
\end{gathered}
$$

Здесь через $\|\cdot\|$ обозначена норма в пространстве $L_{2}(\Omega)$, а через ||$|\cdot| \mid-$ норма в пространстве $L_{2}(\Gamma)$. Получено полное асимптотическое разложение по степеням малого параметра решения рассматриваемой задачи в случае, когда $0<\beta<3 / 2$.

Ключевые слова: сингулярные задачи, оптимальное управление, краевые задачи для систем уравнений в частных производных, асимптотические разложения.

A. R. Danilin. Asymptotics of a solution to a problem of optimal boundary control with two small cosubordinate parameters.

We consider a problem of optimal boundary control for solutions of an elliptic type equation in a bounded domain with smooth boundary with a small coefficient at the Laplace operator, a small coefficient, cosubordinate with the first, at the boundary condition, and integral constraints on the control:

$$
\left\{\begin{array}{l}
\mathcal{L}_{\varepsilon}:=-\varepsilon^{2} \Delta z+a(x) z=f(x), \quad x \in \Omega, \quad z \in H^{1}(\Omega), \\
l_{\varepsilon, \beta} z:=\varepsilon^{\beta} \frac{\partial z}{\partial n}=g(x)+u(x), \quad x \in \Gamma, \\
J(u):=\left\|z-z_{d}\right\|^{2}+\nu^{-1}\|u\| \|^{2} \rightarrow \text { inf }, \quad u \in \mathcal{U},
\end{array}\right.
$$

where $0<\varepsilon \ll 1, \beta \geqslant 0, \beta \in \mathbb{Q}, \nu>0, H^{1}(\Omega)$ is the Sobolev function space, $\partial z / \partial n$ is the derivative of $z$ at the point $x \in \Gamma$ in the direction of the outer (with respect to the domain $\Omega$ ) normal,

$$
\begin{gathered}
a(\cdot), f(\cdot) \in C^{\infty}(\bar{\Omega}), \quad g(\cdot) \in C^{\infty}(\Gamma), \quad \forall x \in \bar{\Omega} \quad a(x) \geqslant \alpha^{2}>0, \\
\mathcal{U}=\mathcal{U}_{1}, \quad \mathcal{U}_{r}:=\left\{u(\cdot) \in L_{2}(\Gamma):\||| u\| \mid \leqslant r\right\} .
\end{gathered}
$$

Here $\|\cdot\|$ and \|\|$\cdot \|$ are the norms in the spaces $L_{2}(\Omega)$ and $L_{2}(\Gamma)$, respectively. We find the complete asymptotic expansion of the solution of the problem in the powers of the small parameter in the case where $0<\beta<3 / 2$.

Keywords: singular problems, optimal control, boundary value problems for systems of partial differential equations, asymptotic expansions.

MSC: 35C20, 35B25, 76M45, 93C70

DOI: $10.21538 / 0134-4889-2020-26-1-102-111$ 


\section{1. Постановка задачи}

Пусть $\Omega \subset \mathbb{R}^{n}(n=2,3)$ - ограниченная область с границей $\Gamma:=\partial \Omega$. Будем предполагать, что $\bar{\Omega}=\Omega \cup \Gamma$ есть многообразие с краем $\Gamma$ класса $C^{\infty}$, расположенное по одну сторону от $\Gamma$.

Рассматривается следующая задача граничного оптимального управления $[1$, гл. 2 , соотношения (2.41), (2.9)]

$$
\left\{\begin{array}{l}
\mathcal{L}_{\varepsilon}:=-\varepsilon^{2} \Delta z+a(x) z=f(x), \quad x \in \Omega, \quad z \in H^{1}(\Omega), \\
l_{\varepsilon, \beta} z:=\varepsilon^{\beta} \frac{\partial z}{\partial n}=g(x)+u(x), \quad x \in \Gamma, \\
J(u):=\left\|z-z_{d}\right\|^{2}+\nu^{-1}\|\| u\|\|^{2} \rightarrow \text { inf, } \quad u \in \mathcal{U},
\end{array}\right.
$$

где $0<\varepsilon \ll 1, \beta \geqslant 0, \beta \in \mathbb{Q}, \nu>0, H^{1}(\Omega)$ - соболевское пространство функций [2;3], $\partial z / \partial n-$ производная функции $z$ в точке $x \in \Gamma$ по направлению внешней (по отношению к области $\Omega$ ) нормали,

$$
\begin{gathered}
a(\cdot), f(\cdot), z_{d}(\cdot) \in C^{\infty}(\bar{\Omega}), \quad g(\cdot) \in C^{\infty}(\Gamma), \quad \forall x \in \bar{\Omega} \quad a(x) \geqslant \alpha^{2}>0, \\
\mathcal{U}=\mathcal{U}_{1}, \quad \mathcal{U}_{r}:=\left\{u(\cdot) \in L_{2}(\Gamma):\||| u \mid\| \leqslant r\right\} .
\end{gathered}
$$

Здесь через ||$|\cdot|||$ обозначена норма в пространстве $L_{2}(\Gamma)$. Скалярное произведение в $L_{2}(\Gamma)$ будем обозначать через $\langle\cdot, \cdot\rangle$. В пространстве $L_{2}(\Omega)$ для нормы и скалярного произведения используются обозначения $\|\cdot\|$ и $(\cdot, \cdot)$ соответственно.

Исследование задач оптимального управления, определяемых уравнениями в частных производных, не теряет своей актуальности (см., например, [4-6] и библиографию в них).

В данной работе рассматривается обобщение задач из работ $[7 ; 8](\beta=2)$ и [9] $(\beta=0)$.

Другие сингулярные задачи оптимального управления решениями эллиптических уравнений рассматривались в $[10 ; 11]$.

Умножив граничное условие в (1.1) на $\varepsilon^{2-\beta}$, получим задачу стандартного вида (см. [1,

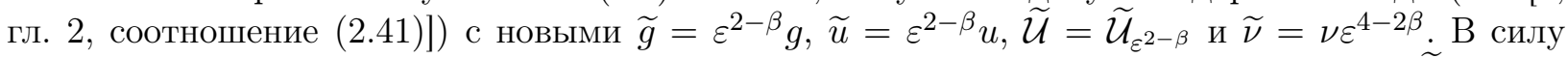
$[1$, гл. 2 , соотношения $(2.41),(2.36),(2.49)]$ и [7, лемма 1 , соотношение $(2.5)]$ найдется $\widetilde{\lambda}_{\varepsilon}$ такое, что единственное оптимальное управление в получившейся задаче и соответствующее ему $z_{\varepsilon}(\cdot)$ находятся как единственное решение следующей задачи:

$$
\begin{aligned}
& \widetilde{u}_{\varepsilon}(\cdot)=\left.\widetilde{\lambda}_{\varepsilon} p_{\varepsilon}\right|_{\Gamma}, \\
& \left\{\begin{array}{lll}
\mathcal{L}_{\varepsilon} z_{\varepsilon}=f(x), & \mathcal{L}_{\varepsilon} p_{\varepsilon}-z_{\varepsilon}=-z_{d}(x), & z_{\varepsilon}, p_{\varepsilon} \in H^{1}(\Omega), \\
\varepsilon^{2} \frac{\partial z_{\varepsilon}}{\partial n}+\widetilde{\lambda}_{\varepsilon} p_{\varepsilon}(x)=\widetilde{g}(x), & \varepsilon^{2} \frac{\partial p_{\varepsilon}}{\partial n}=0, & x \in \Gamma,
\end{array}\right. \\
& \widetilde{\lambda}_{\varepsilon} \in(0 ; \widetilde{\nu}]:\left(\widetilde{\lambda}_{\varepsilon}||\left|p_{\varepsilon}\right||| \leqslant \varepsilon^{2-\beta}\right) \wedge\left(\left(\widetilde{\nu}-\widetilde{\lambda}_{\varepsilon}\right)\left(\varepsilon^{2-\beta}-\widetilde{\lambda}_{\varepsilon}||\left|p_{\varepsilon}\right|||\right)=0\right) \text {. }
\end{aligned}
$$

Обозначив $\lambda_{\varepsilon}:=\varepsilon^{\beta-2} \widetilde{\lambda}_{\varepsilon}$ и вернувшись к исходным переменным, получим (1.4) и (1.5) в виде

$$
\begin{aligned}
& \left\{\begin{array}{lll}
\mathcal{L}_{\varepsilon} z_{\varepsilon}=f(x), & \mathcal{L}_{\varepsilon} p_{\varepsilon}-z_{\varepsilon}=-z_{d}(x), & z_{\varepsilon}, p_{\varepsilon} \in H^{1}(\Omega), \\
l_{\varepsilon, \beta} z_{\varepsilon}+\lambda_{\varepsilon} p_{\varepsilon}(x)=g(x), & l_{\varepsilon, \beta} p_{\varepsilon}=0, & x \in \Gamma,
\end{array}\right. \\
& u_{\varepsilon}(\cdot)=\left.\lambda_{\varepsilon} p_{\varepsilon}\right|_{\Gamma}, \quad \lambda_{\varepsilon} \in\left(0 ; \nu \varepsilon^{2-\beta}\right]:\left(\lambda_{\varepsilon}||\left|p_{\varepsilon}\right| \| \mid \leqslant 1\right) \wedge\left(\left(\nu \varepsilon^{2-\beta}-\lambda_{\varepsilon}\right)\left(1-\lambda_{\varepsilon}||\left|p_{\varepsilon}\right|||\right)=0\right) .
\end{aligned}
$$


Таким образом, оптимальное управление $u_{\varepsilon}$ и состояние $z_{\varepsilon}$ в задаче $(1.1),(1.2)$ определяются из решения задачи (1.6), (1.7).

В [11, теорема 1] показано, что задача вида

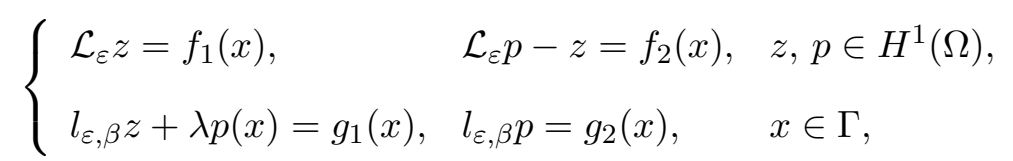

при выполнении условий (1.3) и

$$
f_{1}(\cdot), f_{2}(\cdot) \in C^{\infty}(\bar{\Omega}), \quad g_{1}(\cdot), g_{2}(\cdot) \in C^{\infty}(\Gamma)
$$

разрешима единственным образом при любом $\varepsilon>0$ и $\lambda>0$ и справедливы соотношения $z_{\varepsilon}, p_{\varepsilon} \in C^{\infty}(\bar{\Omega})$.

Цель работы - изучить поведение $z_{\varepsilon}, p_{\varepsilon}$ и $\lambda_{\varepsilon}$ при $\varepsilon \rightarrow 0$ и построить асимптотическое разложение $z_{\varepsilon}, p_{\varepsilon}$ и $\lambda_{\varepsilon}$ при $\varepsilon \rightarrow 0$ с точностью до любой степени параметра $\varepsilon$.

\section{2. Априорные оценки и разрешимость краевых задач}

Отметим, что решение краевой задачи (1.1) понимается в обобщенном смысле (см., например, $\left[1\right.$, гл. $1, \S 3$, п. 3.4]): для любого $\varphi \in H^{1}(\Omega)$ справедливо равенство

$$
\varepsilon^{2}\left(\nabla z_{\varepsilon}, \nabla \varphi\right)+\left(a(\cdot) z_{\varepsilon}, \varphi\right)-\varepsilon^{2-\beta}\left\langle g+u_{\varepsilon}, \varphi\right\rangle=(f, \varphi) .
$$

Как и в [7], для получения априорных оценок используются априорные оценки для эллиптических операторов [3, гл. 2 , теорема $5.1 ; 12$, гл. III, формула (1.11)] и частный случай неравенства Эрлинга

$$
\|u\| \|^{2} \leqslant K\left(\delta^{-1}\|u\|^{2}+\delta\|\nabla u\|^{2}\right), \quad 0<\delta \leqslant \delta_{0}
$$

(см., например, [13, гл. XIV, $\S 3,(3.3) ; 14$, гл. I, $\S 6,(6.19)])$. Отметим, что $K$ в $(2.2)$ зависит от $\delta_{0}$.

В дальнейшем различные положительные константы, зависящие только от области $\Omega$, коэффициента $a(\cdot)$ и неизменяемых величин (например, $\delta_{0}$ ), часто будем обозначать одной и той же буквой $-K$ или $C$.

Лемма 1. Пусть выполнены условия (1.3) и (1.9), а z, p-решение задачи (1.8). Тогда

$$
\|z\|^{2}+\left.\lambda \varepsilon^{2-\beta}\||| p\|\right|^{2}=\left(f_{1}, p\right)-\left(f_{2}, z\right)+\varepsilon^{2-\beta}\left\langle g_{1}, p\right\rangle-\varepsilon^{2-\beta}\left\langle g_{2}, z\right\rangle .
$$

Д о к а з а т е л ь с т в о. В силу (2.1) - определения обобщенного решения задачи (1.8) для $z, p \in H^{1}(\Omega)$ справедливы равенства

$$
\begin{aligned}
& \varepsilon^{2}(\nabla z, \nabla p)+(a(\cdot) z, p)+\varepsilon^{2-\beta}\left\langle\lambda p-g_{1}, p\right\rangle=\left(f_{1}, p\right), \\
& \varepsilon^{2}(\nabla p, \nabla z)+(a(\cdot) p, z)-(z, z)-\varepsilon^{2-\beta}\left\langle g_{2}, z\right\rangle=\left(f_{2}, z\right) .
\end{aligned}
$$

Вычитая из первого равенства второе, получим (2.3).

Лемма 2. Пусть выполнено условие (1.3), $\bar{f} \in L_{2}(\Omega), q \in L_{2}(\Gamma)$ и $z_{\varepsilon}$ есть решение задачи

$$
\mathcal{L}_{\varepsilon} z_{\varepsilon}=\bar{f}(x), \quad x \in \Omega, \quad z_{\varepsilon} \in H^{1}(\Omega), \quad l_{\varepsilon, \beta} z_{\varepsilon}=q(x), \quad x \in \Gamma .
$$

Тогда существует $K>0$ такое, что

$$
\max \left\{\left\|z_{\varepsilon}\right\|, \varepsilon^{1 / 2}\left\|\left|z_{\varepsilon}\|\mid, \varepsilon\| \nabla z_{\varepsilon} \|\right\} \leqslant K\left(\|\bar{f}\|+\varepsilon^{3 / 2-\beta}\|\mid q\| \|\right)=: K \cdot D(\bar{f}, q ; \varepsilon, \beta) .\right.\right.
$$


Д о к а з а т е л ь с т в о. В силу (2.1) и условий (1.3) для $z_{\varepsilon}$ - решения задачи (2.4) получим

$$
\begin{aligned}
& \varepsilon^{2}\left\|\nabla z_{\varepsilon}\right\|^{2}+\alpha^{2}\left\|z_{\varepsilon}\right\|^{2} \leqslant\left\|z_{\varepsilon}\right\| \cdot\|\bar{f}\|+\varepsilon^{2-\beta}\left\|\left|\|q\| \cdot\left\|z_{\varepsilon} \mid\right\|\right.\right. \\
& \stackrel{(2.2)}{\leqslant}\left\|z_{\varepsilon}\right\|\left(\|\bar{f}\|+K \delta^{-1} \varepsilon^{2-\beta}\|\mid q\| \|\right)+\left\|\nabla z_{\varepsilon}\right\| K \delta \varepsilon^{2-\beta}\|\mid q\| \| .
\end{aligned}
$$

Решая данное квадратичное неравенство относительно величин $\left\|z_{\varepsilon}\right\|$ и $\left\|\nabla z_{\varepsilon}\right\|$, имеем

$$
\begin{aligned}
& \left\|z_{\varepsilon}\right\| \leqslant \frac{\|\bar{f}\|+K \delta^{-1} \varepsilon^{2-\beta} \mid\|q\| \|}{\alpha^{2}}+\frac{K \delta \varepsilon^{2-\beta} \mid\|q\| \|}{2 \alpha \varepsilon}, \\
& \left\|\nabla z_{\varepsilon}\right\| \leqslant \frac{K \delta \varepsilon^{2-\beta} \mid\|q\| \|}{\varepsilon^{2}}+\frac{\|\bar{f}\|+K \delta^{-1} \varepsilon^{2-\beta} \mid\|q\| \|}{2 \alpha \varepsilon} .
\end{aligned}
$$

При $\delta=\varepsilon^{1 / 2}$ из (2.6) получим (2.5).

Теорема 1. Пусть выполнены условия (1.3) и (1.9). Если z, p-решения задачи (1.8), то существует $K>0$ такое, что справедливы оценки

$$
\begin{aligned}
& \max \left\{\|z\|, \varepsilon^{1 / 2}\|z \mid\|, \varepsilon\|\nabla z\|\right\} \leqslant K\left(1+\lambda \varepsilon^{1-\beta}\right) \widetilde{D}\left(f_{1}, f_{2}, g_{1}, g_{2} ; \varepsilon, \beta\right), \\
& \max \left\{\|p\|, \varepsilon^{1 / 2}\|p\|\|, \varepsilon\| \nabla p \|\right\} \leqslant K\left(1+\lambda \varepsilon^{1-\beta}\right) \widetilde{D}\left(f_{1}, f_{2}, g_{1}, g_{2} ; \varepsilon, \beta\right),
\end{aligned}
$$

где $\widetilde{D}\left(f_{1}, f_{2}, g_{1}, g_{2} ; \varepsilon, \beta\right):=\left\|f_{1}\right\|+\left\|f_{2}\right\|+\varepsilon^{3 / 2-\beta}\left(\left|\left\|g_{1}\left|\left\|+\left|\left\|g_{2} \mid\right\|\right)\right.\right.\right.\right.\right.$.

Д о к а з а т е л ь с т в о. Представим $z$ и $p$ в виде $z=z_{1}+z_{2}$ и $p=p_{1}+p_{2}$, где

$$
\begin{gathered}
\mathcal{L}_{\varepsilon} z_{1}=f_{1}, \quad \mathcal{L}_{\varepsilon} p_{1}=z_{1}+f_{2}, \quad l_{\varepsilon, \beta} z_{1}=g_{1}, \quad l_{\varepsilon, \beta} p_{1}=g_{2}, \\
\mathcal{L}_{\varepsilon} z_{2}=0, \quad \mathcal{L}_{\varepsilon} p_{2}-z_{2}=0, \quad l_{\varepsilon, \beta} z_{2}+\lambda p_{2}=-\lambda p_{1}, \quad l_{\varepsilon, \beta} p_{2}=0 .
\end{gathered}
$$

Для $z_{1}$ и $p_{1}$ согласно (2.5) справедливы неравенства

$$
D\left(f_{1}, g_{1} ; \varepsilon, \beta\right) \leqslant \widetilde{D}\left(f_{1}, f_{2}, g_{1}, g_{2} ; \varepsilon, \beta\right)
$$

и

$$
D\left(f_{2}+z_{1}, g_{2} ; \varepsilon, \beta\right) \leqslant \widetilde{D}\left(f_{1}, f_{2}, g_{1}, g_{2} ; \varepsilon, \beta\right) .
$$

В силу (2.3) для $z_{2}$ и $p_{2}$ имеем

$$
\left\|z_{2}\right\|^{2}+\lambda \varepsilon^{2-\beta}\left\|\left|p_{2}\right|\right\|^{2}=\varepsilon^{2-\beta}\left\langle-\lambda p_{1}, p_{2}\right\rangle .
$$

Отсюда $\left.\lambda \varepsilon^{2-\beta}||\left|p_{2}\right|\right|^{2} \leqslant \lambda \varepsilon^{2-\beta}||\left|p_{1}\right||| \cdot||\left|p_{2}\right|||$, т. е. ||$\left|p_{2}\right||| \leqslant||\left|p_{1}\right|||$.

Поэтому

$$
D\left(0, \lambda\left(p_{1}+p_{2}\right) ; \varepsilon, \beta\right) \leqslant 2 \lambda \varepsilon^{3 / 2-\beta}||\left|p_{1}\right||| \leqslant 2 \lambda \varepsilon^{3 / 2-\beta} \varepsilon^{-1 / 2} \widetilde{D}\left(f_{1}, f_{2}, g_{1}, g_{2} ; \varepsilon, \beta\right),
$$

а $D\left(z_{2}, 0 ; \varepsilon, \beta\right) \leqslant\left\|z_{2}\right\|$. Наконец, вследствие неравенства треугольника выводим оценки (2.7).

Утверждение 1. Пусть $а(\cdot), f(\cdot)$ и $g(\cdot)$ удовлетворяют условиям (1.3).

Если $z_{\varepsilon}(\cdot), p_{\varepsilon}(\cdot) \in C^{\infty}(\bar{\Omega})$ есть решение задачи (1.6), (1.7), то при $\varepsilon \rightarrow 0$ справедливы следующие асимптотические представления:

$$
\begin{aligned}
& \left\|z_{\varepsilon}\right\|=O(1)+O\left(\varepsilon^{3 / 2-\beta}\right), \quad\left\|z_{\varepsilon}\right\|==O\left(\varepsilon^{-1 / 2}\right)+O\left(\varepsilon^{1-\beta}\right), \\
& \left\|\nabla z_{\varepsilon}\right\|=O\left(\varepsilon^{-1}\right)+O\left(\varepsilon^{1 / 2-\beta}\right), \quad\left\|p_{\varepsilon}\right\|=O(1)+O\left(\varepsilon^{3 / 2-\beta}\right), \\
& \left\|p_{\varepsilon}\right\|=O\left(\varepsilon^{-1 / 2}\right)+O\left(\varepsilon^{1-\beta}\right), \quad\left\|\nabla p_{\varepsilon}\right\|=O\left(\varepsilon^{-1}\right)+O\left(\varepsilon^{1 / 2-\beta}\right) .
\end{aligned}
$$


Д о к а з а т е л ь с т в о. В силу того что $\lambda_{\varepsilon}\left\|p_{\varepsilon}\right\| \mid \leqslant 1$ для $z_{\varepsilon}$, имеем

$$
D\left(f,-\lambda p_{\varepsilon}+g ; \varepsilon, \beta\right) \leqslant\|f\|+\varepsilon^{3 / 2-\beta}\left(\left|\left\|p_{\varepsilon}\right\|\right|+1\right)=O(1)+O\left(\varepsilon^{3 / 2-\beta}\right) ;
$$

это согласно (2.5) доказывает асимптотические оценки для $z_{\varepsilon}$. Теперь для $p_{\varepsilon}$ получим

$$
D\left(z_{\varepsilon}-z_{d}, 0 ; \varepsilon, \beta\right) \leqslant\left\|z_{d}\right\|+\left\|z_{\varepsilon}\right\|=O(1)+O\left(\varepsilon^{3 / 2-\beta}\right)
$$

это согласно (2.5) доказывает асимптотические оценки для $p_{\varepsilon}$.

В силу (2.8) и того, что $\lambda_{\varepsilon}=O\left(\varepsilon^{2-\beta}\right)$ (см. (1.7)), выводим $\lambda_{\varepsilon}\left\|p_{\varepsilon}\right\| \|=O\left(\varepsilon^{3 / 2-\beta}\right)+O\left(\varepsilon^{3-2 \beta}\right)$.

Тем самым если $3>2 \beta$, то $\lambda_{\varepsilon}\left\|\left|p_{\varepsilon} \|\right|=o(1)\right.$ при $\varepsilon \rightarrow 0$ и, следовательно, в силу (1.7) $\lambda_{\varepsilon}=\nu \varepsilon^{2-\beta}$.

В дальнейшем будем считать, что

$$
\frac{3}{2}>\beta, \quad \lambda_{\varepsilon}=\nu \varepsilon^{2-\beta} .
$$

В этом случае справедлива следующая теорема аппроксимации для $z_{\varepsilon}$ и $p_{\varepsilon}$.

Теорема 2. Пусть функиии $f_{1, \varepsilon, m}(\cdot), f_{2, \varepsilon, m}(\cdot) \in C^{\infty}(\bar{\Omega}), g_{1, \varepsilon, m}(\cdot), g_{2, \varepsilon, m}(\cdot) \in C^{\infty}(\bar{\Gamma})$ u въполнены условия (1.3) и (2.9). Если

$$
\max \left\{\left\|f_{i, \varepsilon, m}\right\|,\left\|\mid g_{i, \varepsilon, m}\right\| \|: i=1,2\right\}=O\left(\varepsilon^{m}\right), \quad \varepsilon \rightarrow 0,
$$

a $z_{m}, p_{m}$ - решение задачи

$$
\left\{\begin{array}{l}
\mathcal{L}_{\varepsilon} z_{m}=f(x)+f_{1, \varepsilon, m}(x), \quad \mathcal{L}_{\varepsilon} p_{m}+z_{m}=-z_{d}(x)+f_{2, \varepsilon, m}(x), \quad z_{m}, p_{m} \in H^{1}(\Omega), \\
l_{\varepsilon, \beta} z_{m}+\nu \varepsilon^{2-\beta} p_{m}(x)=g(x)+g_{1, \varepsilon, m}(x), \quad l_{\varepsilon, \beta} p_{m}=g_{2, \varepsilon, m}(x), \quad x \in \Gamma,
\end{array}\right.
$$

то для $z_{\varepsilon, m}:=z_{\varepsilon}-z_{m}$ и $p_{\varepsilon, m}:=p_{\varepsilon}-p_{m}$, где $z_{\varepsilon}, p_{\varepsilon}$, - решение задачи (1.6), (2.9), справедливь следующие асимптотические оченки:

$$
\max \left\{\left\|z_{\varepsilon, m}\right\|, \varepsilon^{1 / 2}\left|\left\|z_{\varepsilon, m} \mid\right\|, \varepsilon\left\|\nabla z_{\varepsilon, m}\right\|,\left\|p_{\varepsilon, m}\right\|, \varepsilon^{1 / 2}\left\|p_{\varepsilon, m}\right\|\|, \varepsilon\| \nabla p_{\varepsilon, m} \|\right\}=O\left(\varepsilon^{m}\right), \quad \varepsilon \rightarrow 0 .\right.
$$

Д о к а з а т е л ь с т в о. Функции $z_{\varepsilon, m}$ и $p_{\varepsilon, m}$ удовлетворяют системе вида (2.10) с правыми частями порядка $O\left(\varepsilon^{m}\right)$. В силу теоремы 1 с учетом соотношения $(2.9)$ получим

$$
\widetilde{D}\left(f_{1, \varepsilon, m}, f_{2, \varepsilon, m}, g_{1, \varepsilon, m}, g_{2, \varepsilon, m} ; \varepsilon, \beta\right)=O\left(\varepsilon^{m}\right)+O\left(\varepsilon^{m}\right)+\varepsilon^{3 / 2-\beta}\left(O\left(\varepsilon^{m}\right)+O\left(\varepsilon^{m}\right)\right)=O\left(\varepsilon^{m}\right) \text {. }
$$

Отметим, что вследствие гладкости коэффициентов всех разложений из априорных оценок Шаудера (см., напрмер, [3, гл. 2, теорема 5.1]) и теормы вложения Соболева [2, гл. I, п. 8, теорема 1] следует, что соотношения (2.11) справедливы и в равномерной норме.

\section{3. Построение асимптотики}

В силу теоремы 2 для построения асимптотического разложения решения рассматриваемой задачи нужно построить его формальное асимптотическое решение (ф. а. р.) (см., например, $[15$, с. 10]), которое осуществляется аналогично тому, как это делается в случае одного уравнения $[16 ; 17]$.

Внешнее разложение решения ищем в виде рядов

$$
z_{\text {out }}(x, \varepsilon)=\sum_{k=0}^{\infty} \varepsilon^{2 k} z_{k}(x), \quad p_{\text {out }}(x, \varepsilon)=\sum_{k=0}^{\infty} \varepsilon^{2 k} p_{k}(x), \varepsilon \rightarrow 0 .
$$


Коэффициенты $z_{k}(x), p_{k}(x)$ находятся из соответствующей рекуррентной системы

$$
\begin{cases}z_{0}(x)=-\frac{f(x)}{a(x)}, \quad p_{0}(x)=\frac{z_{0}(x)-z_{d}(x)}{a(x)}, & \\ z_{2 k}(x)=\frac{\Delta z_{2 k-2}}{a(x)}, & p_{2 k}=\frac{\Delta p_{2 k-2}+z_{2 k}}{a(x)}, \quad k \geqslant 1 .\end{cases}
$$

Все $z_{2 k}(x), p_{2 k}(x) \in C^{\infty}(\bar{\Omega})$, но не удовлетворяют граничным условиям.

Для того чтобы устранить невязку в граничных условиях, построим экспоненциально убывающие функции в окрестности всей границы $\Gamma$, удовлетворяющие соответствующей однородной системе.

С учетом гладкости $Г$ в ее малой окрестности можно ввести систему координат $(s ; \tau)$, где $s$ - это координаты на $\Gamma$, а $\tau-$ расстояние от текущей точки $x \in \Omega$ до $\Gamma$.

Пограничный слой имеет ширину порядка $\varepsilon$, а поправочные функции (внутреннее разложение) нужны не во всей области $\Omega$, а лишь в ее малой окрестности. Поэтому после построения поправочные функции необходимо умножить на срезающую функцию $\eta$, т. е. функцию с носителем в малой окрестности границы и равной тождественно 1 в некоторой меньшей окрестности границы.

В пограничном слое перейдем к новым, растянутым, координатам (см., например, [15, c. $31-34]) \xi=\tau \varepsilon^{-1}$.

При этом оператор $\mathcal{L}_{\varepsilon}$ перейдет в оператор

$$
\widetilde{\mathcal{L}}_{\varepsilon} Z=-\frac{\partial^{2}}{\partial \xi^{2}} Z-\varepsilon L_{1} \frac{\partial}{\partial \xi} Z-\varepsilon^{2} L_{2} Z+\widetilde{a}(s, \varepsilon \xi) Z=:-\frac{\partial^{2}}{\partial \xi^{2}} Z+\widetilde{a}(s, \varepsilon \xi) Z+\varepsilon \mathcal{M}_{\varepsilon} Z .
$$

Здесь $L_{1}$ и $L_{2}$ - дифференциальные операторы 1-го и 2-го порядка, содержащие лишь дифференцирование по переменной $s$, с гладкими коэффициентами от $s$ и $\tau=\varepsilon \xi$, а $\widetilde{a}(s, \tau)-$ это функция $a(x)$ в переменных $s, \tau$.

Таким образом, однородная система для функций пограничного слоя в переменных $s$ и $\xi$, соответствующая системе из (1.4), имеет вид

$$
\left\{\widetilde{\mathcal{L}}_{0, \varepsilon} Z:=-\frac{\partial^{2}}{\partial \xi^{2}} Z+\widetilde{a}(s, \varepsilon \xi) Z=-\varepsilon \mathcal{M}_{\varepsilon} Z, \quad \widetilde{\mathcal{L}}_{0, \varepsilon} P-Z=-\varepsilon \mathcal{M}_{\varepsilon} P .\right.
$$

Для граничных условий, с учетом того что

$$
\frac{\partial}{\partial n} Z(s, \tau / \varepsilon)=-\frac{\partial}{\partial \tau} Z(s, \tau / \varepsilon)=-\varepsilon^{-1} \frac{\partial}{\partial \xi} Z(s, \xi),
$$

выводим следующие соотношения:

$$
\left\{\begin{array}{l}
-\varepsilon^{\beta-1} \frac{\partial}{\partial \xi} Z(s, 0)+\varepsilon^{\beta} \frac{\widetilde{\partial}}{\partial n} z_{\text {out }}(s, 0)+\nu \varepsilon^{2-\beta}\left(P(s, 0)+\widetilde{p_{\text {out }}}(s, 0)\right) \stackrel{\text { as }}{=} \widetilde{g}(s), \\
-\varepsilon^{\beta-1} \frac{\partial}{\partial \xi} P(s, 0)+\varepsilon^{\beta} \frac{\widetilde{\partial}}{\partial n} p_{\text {out }}(s, 0) \stackrel{\text { as }}{=} 0 .
\end{array}\right.
$$

Здесь волна над функцией, определенной в переменных $x$, означает выражение этой функции в переменных $s$ и $\tau$.

Система (3.4) показывает, что если $Z$ имеет порядок $\gamma$ (т. е. $Z=O\left(\varepsilon^{\gamma}\right)$ ), то и $P$ имеет тот же порядок. В этом случае порядки слагаемых в (3.5) таковы: $\beta-1+\gamma, \beta, 2-\beta+\gamma$ и $2-\beta$. Главное слагаемое должно иметь порядок 0 . В силу условий (2.9) это $\beta-1+\gamma$. Тем самым $\gamma=1-\beta$. Последнее соотношение определяет вид разложения в пограничном слое. 
Пусть $\beta=\widetilde{n} / \widetilde{m}-$ несократимая дробь. Тогда

$$
Z_{i n}(s, \xi, \varepsilon)=\varepsilon^{1-\beta} \sum_{m=0}^{\infty} \varepsilon^{m / \widetilde{m}} Z_{m}(s, \xi), \quad P_{i n}(s, \xi, \varepsilon)=\varepsilon^{1-\beta} \sum_{m=0}^{\infty} \varepsilon^{m / \widetilde{m}} P_{m}(s, \xi) .
$$

Подставляя ряды (3.6) в систему (3.4) и разлагая коэффициенты в уравнениях системы и операторов $\widetilde{\mathcal{L}}_{0, \varepsilon}$ и $\mathcal{M}_{\varepsilon}$, определенных в $(3.3)$, в ряды Тейлора по переменной $\tau=\varepsilon \xi$, получим следующую систему:

$$
\begin{cases}\widetilde{\mathcal{L}}_{0} Z_{0}:=-\frac{\partial^{2}}{\partial \xi^{2}} Z_{0}+\widetilde{a}_{0}(s) Z_{0}=0, & \widetilde{\mathcal{L}}_{0} P_{0}+Z_{0}=0, \\ \widetilde{\mathcal{L}}_{0} Z_{m}=F_{m}(s, \xi), & \widetilde{\mathcal{L}}_{0} P_{m}+Z_{m}=G_{m}(s, \xi), \quad m>0,\end{cases}
$$

где $F_{m}(s, \xi)$ и $G_{m}(s, \xi)$ линейно выражаются через предыдущие функции $Z_{k}, P_{k}$ и их производные и полиномиально зависят от $\xi$ и гладко от $s$, а функция

$$
a(x)=\widetilde{a}(s, \varepsilon \xi)=\sum_{i=0}^{\infty} \varepsilon^{i} \xi^{i} \widetilde{a}_{i}(s)
$$

разложена в ряд по степеням малого параметра.

Подстановка соответствующих рядов в граничные условия приведет к следующим системам:

$$
\begin{cases}-\frac{\partial}{\partial \xi} Z_{0}(s, 0)=\widetilde{g}(s), & -\frac{\partial}{\partial \xi} P_{0}(s, 0)=0 \\ -\frac{\partial}{\partial \xi} Z_{m}(s, 0)=\widetilde{g}_{1, m}(s), & -\frac{\partial}{\partial \xi} P_{m}(s, 0)=\widetilde{g}_{2, m}(s), \quad m>1\end{cases}
$$

где функции $g_{m}(\cdot), q_{m}(\cdot)$ определяются внешним разложением, функциями $Z_{k}, P_{k}$ при $k<m$, при этом $Z_{m}$ и $P_{m}$ должны экспоненциально убывать при $\xi \rightarrow+\infty$.

Таким образом, задачи для нахождения $Z_{m}$ и $P_{m}$ имеют следующий вид:

$$
\begin{cases}\widetilde{\mathcal{L}}_{0} Z=F, & \widetilde{\mathcal{L}}_{0} P+Z=G \\ -\frac{\partial}{\partial \xi} Z(s, 0)=\widetilde{g}_{1}(s), & -\frac{\partial}{\partial \xi} P(s, 0)=\widetilde{g}_{2}(s)\end{cases}
$$

Как хорошо известно, первое уравнение из (3.9) имеет нужное нам решение вида

$$
Z(s, \xi)=C_{1}(s) e^{-\sqrt{\widetilde{a}_{0}(s)} \xi}+\widehat{Z}(s, \xi),
$$

где $C_{1}(s)$ - функция, подлежащая определению, а $\widehat{Z}(s, \xi)$ - какое-нибудь частное решение уравнения $\widetilde{\mathcal{L}}_{0} Z=F$. При этом если

$$
F(s, \xi)=e^{-\sqrt{\tilde{a}_{0}(s) \xi}} Q_{l}(s, \xi),
$$

где $Q_{l}(s, \xi)$ - полином степени $l$ по $\xi$ с коэффициентами, зависящими от $s$, то

$$
\widehat{Z}(s, \xi)=e^{-\sqrt{\widetilde{a}_{0}(s)} \xi} \xi Q_{l, 1}(s, \xi) .
$$

Здесь $Q_{l, 1}(s, \xi)$ - тоже полином степени $l$ по $\xi$ с коэффициентами, зависящими от $s$, однозначно определяемый по $Q_{l}(s, \xi)$.

Теперь второе уравнение из (3.9) в силу (3.10) можем записать как

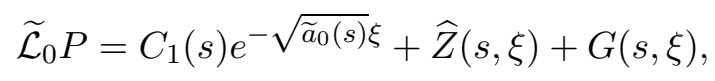


а любое, экспоненциально убывающее при $\xi \rightarrow+\infty$ решение этого уравнения определяется формулой

$$
P(s, \xi)=C_{2}(s) e^{-\sqrt{\widetilde{a}_{0}(s)} \xi}+C_{1}(s) \frac{\xi}{2 \sqrt{\widetilde{a}_{0}(s)}} e^{-\sqrt{\widetilde{a}_{0}(s)} \xi}+\widehat{P}(s, \xi),
$$

где $\widehat{P}(s, \xi)$ - какое-нибудь частное решение уравнения $\widetilde{\mathcal{L}}_{0} P=\widehat{Z}(s, \xi)+G(s, \xi)$.

Неизвестные функции $C_{1}(s)$ и $C_{2}(s)$ определятся из граничных условий задачи $(3.9)-$ они (в силу (3.10) и (3.11)) являются решением системы уравнений

$$
\left\{\begin{array}{l}
-\frac{\partial}{\partial \xi} Z(s, 0)=\sqrt{\widetilde{a}_{0}(s)} C_{1}(s)-\frac{\partial}{\partial \xi} \widehat{Z}(s, 0)=\widetilde{g}_{1}(s), \\
-\frac{\partial}{\partial \xi} P(s, 0)=\sqrt{\widetilde{a}_{0}(s)} C_{2}(s)-C_{1}(s) \frac{1}{2 \sqrt{\widetilde{a}_{0}(s)}}-\frac{\partial}{\partial \xi} \widehat{P}(s, 0)=\widetilde{g}_{2}(s),
\end{array}\right.
$$

из которой получим

$$
C_{1}(s)=\frac{\widetilde{g}_{1}(s)+\frac{\partial}{\partial \xi} \widehat{Z}(s, 0)}{\sqrt{\widetilde{a}_{0}(s)}}, \quad C_{2}(s)=\frac{\widetilde{g}_{2}(s)+\frac{\partial}{\partial \xi} \widehat{P}(s, 0)+\frac{C_{1}(s)}{2 \sqrt{\widetilde{a}_{0}(s)}}}{\sqrt{\widetilde{a}_{0}(s)}}
$$

Таким образом, задача (3.7), (3.8) при каждом $m \geqslant 0$ имеет единственное экспоненциально убывающее решение решение $\left\{Z_{m}, P_{m}\right\}$.

Теперь ряды

$$
\begin{aligned}
& z_{i t}=\sum_{k=0}^{\infty} \varepsilon^{2 k} z_{k}(x)+\eta(s, \tau) \varepsilon^{1-\beta} \sum_{m=0}^{\infty} \varepsilon^{m / \widetilde{m}} Z_{m}(s, \xi) \\
& p_{i t}=\sum_{k=0}^{\infty} \varepsilon^{2 k} p_{k}(x)+\eta(s, \tau) \varepsilon^{1-\beta} \sum_{m=0}^{\infty} \varepsilon^{m / \widetilde{m}} P_{m}(s, \xi),
\end{aligned}
$$

где $x$ и $\tau, s$ связаны введенной системой координат, хорошо аппроксимируют всю задачу (1.6), (2.9). Поэтому справедлива следующая основная теорема.

Теорема 3. Пусть выполнены условия (1.3) и (2.9). Тогда ряды (3.12), коэффичиенты которых для рядов (3.1) определяются по формулам (3.2), а для рядов (3.6) - как решения задач (3.7), (3.8), суть равномерные (как в смысле пространства $H^{1}(\Omega)$, так и в смысле пространства $C(\bar{\Omega}))$ асимптотические разложения при $\varepsilon \rightarrow 0$ функиий $z_{\varepsilon}(x)$ и $p_{\varepsilon}(x)-$ решения задачи (1.6), (2.9).

\section{СПИСОК ЛИТЕРАТУРЫ}

1. Лионс Ж.-Л. Оптимальное управление системами, описываемыми уравнениями с частными производными. М.: Мир, 1972. 414 с.

2. Соболев С.Л. Некоторые применения функционального анализа в математической физике. Л.: Изд-во ЛГУ, 1950. 255 с.

3. Лионс Ж.-Л., Мадженес Э. Неоднородные граничные задачи и их приложения. М.: Мир, 1971. $371 \mathrm{c.}$

4. Casas E. A review on sparse solutions in optimal control of partial differential equations // SeMA J. 2017. Vol. 74. P. 319-344. doi: 10.1007/s40324-017-0121-5.

5. Lou H., Yong J. Second-order necessary conditions for optimal control of semilinear elliptic equations with leading term containing controls // Math. Control Relat. Fields. 2018. Vol. 8, no. 1. P. 57-88. doi: $10.3934 /$ mcrf.2018003.

6. Betz Livia M. Second-order sufficient optimality conditions for optimal control of nonsmooth, semilinear parabolic equations // SIAM J. Control Optim. 2019. Vol. 57, no. 6. P. 4033-4062.

doi: 10.1137/19M1239106 . 
7. Данилин А.P., Зорин А.П. Асимптотика решения задачи оптимального граничного управления // Тр. Ин-та математики и механики УрО РАН. 2009. Т. 15, № 4. С. 95-107.

8. Данилин А.Р., Зорин А.П. Асимптотическое разложение решения задачи оптимального граничного управления // Докл. АН. 2011. Т. 440, № 4. С. 1-4.

9. Зорин А.П. Асимптотическое разложение решения задачи оптимального управления ограниченным потоком на границе // Тр. Ин-та математики и механики УрО РАН. 2013. Т. 19, № 1. C. $115-121$.

10. Капустян В.Е. Асимптотика ограниченных управлений в оптимальных эллиптических задачах // Докл. АН Украины. Сер. Математика, естествознание, технические науки. 1992. № 2. С. 70-74.

11. Данилин А.P. Оптимальное граничное управление в области с малой полостью // Уфим. мат. журн. 2012. Т. 4, № 2. С. 87-100.

12. Ладыженская О.А., Уральцева Н.Н. Линейные и квазилинейные уравнения эллиптического типа. М.: Наука, 1964. 540 с.

13. Морен К. Методы гильбертова пространства. М.: Мир, 1965. 570 с.

14. Ладыженская О.А. Краевые задачи математической физики. М.: Наука, 1973. 407 с.

15. Ильин А.М. Согласование асимптотических разложений решений краевых задач. М.: Наука, 1989. $336 \mathrm{c.}$

16. Вишик М.И., Люстерник Л.А. Регулярное вырождение и пограничный слой для линейных дифференциальных уравнений с малым параметром // Успехи мат. наук. 1957. Т.12, вып. 5. С.3122.

17. Ильин А.М. Пограничный слой // Современные проблемы математики. Фундаментальные направления. Т. 34. М.: ВНИТИ, 1988. С. 175-214. (Итоги науки и техники ВИНИТИ АН СССР.)

Поступила 4.11.2019

После доработки 10.01.2020

Принята к публикации 14.01.2020

Данилин Алексей Руфимович

д-р физ.-мат. наук, профессор,

зав. отделом

Институт математики и механики им. Н. Н. Красовского УрО РАН

г. Екатеринбург

e-mail: dar@imm.uran.ru

\section{REFERENCES}

1. Lions J.-L. Optimal control of systems governed by partial differential equations. Berlin; New York: Springer-Verlag, 1971, 396 p. ISBN: 9783540051152. Translated to Russian under the title Optimal'noe upravlenie sistemami, opisyvaemymi uravneniyami s chastnymi proizvodnymi. Moscow: Mir Publ., 1972, $414 \mathrm{p}$.

2. Sobolev S.L. Some Applications of Functional Analysis in Mathematical Physics. Providence, RI: Amer. Math. Soc., 1991, 286 p. ISBN: 0-8218-4549-7. Original Russian text (1st ed.) published in Sobolev S.L. Nekotorye primeneniya funktsional'nogo analiza v matematicheskoi fizike. Leningrad: Leningr. Gos. Univ. Publ., 1950, 255 p.

3. Lions J.-L., Magenes E. Non-homogeneous boundary value problems and their applications. Berlin: Springer-Verlag, 1972, 357 p. ISBN: 3540053638. Translated to Russian under the title Neodnorodnye granichnye zadachi i ikh prilozheniya. Moscow: Mir Publ., 1971, 371 p.

4. Casas Eduardo. A review on sparse solutions in optimal control of partial differential equations. SeMA J., 2017, vol. 74, pp. 319-344. doi: 10.1007/s40324-017-0121-5 .

5. Lou H., Yong J. Second-order necessary conditions for optimal control of semilinear elliptic equations with leading term containing controls. Math. Control Relat. Fields, 2018, vol. 8, no. 1, pp. 57-88. doi: $10.3934 /$ mcrf.2018003.

6. Betz Livia M. Second-order sufficient optimality conditions for optimal control of nonsmooth, semilinear parabolic equations. SIAM J. Control Optim., 2019, vol. 57, no. 6, pp. 4033-4062.

doi: $10.1137 / 19 \mathrm{M} 1239106$. 
7. Danilin A.R., Zorin A.P. Asymptotics of a solution to an optimal boundary control problem. Proc. Steklov Inst. Math. (Suppl.), 2010, vol. 269, suppl. 1, pp. S81-S94. doi: 10.1134/S0081543810060088.

8. Danilin A.R., Zorin A.P. Asymptotic expansion of solutions to optimal boundary control problems. Dokl. Math.., 2011, vol. 84, no. 2, pp. 665-668. doi: 10.1134/S106456241106024X.

9. Zorin A.P. Asymptotic expansion of a solution to the problem of optimal control of a bounded flow at a boundary. Trudy Inst. Mat. i Mekh. UrO RAN, 2013, vol. 19, no. 1, pp. 115-120 (in Russian).

10. Kapustyan V.E. Asymptotics of bounded controls in optimal elliptic problems. Dokl. Akad. Nauk Ukrainy, 1992, no. 2, pp. 70-74 (in Russian).

11. Danilin A.R. Optimal boundary control in a small concave domain. Ufimsk. Mat. Zh., 2012, vol. 4, no. 2, pp. 87-100 (in Russian).

12. Ladyzhenskaya O.A., Uraltseva N.N. Linear and quasilinear elliptic equations. N Y; London: Acad. Press, 1968, 495 p. ISBN: 9780080955544. Original Russian text published in Ladyzhenskaya O.A., Uraltseva N.N. Lineinye i kvazilineinye uravneniya ellipticheskogo tipa. Moscow: Nauka Publ., 1964, $540 \mathrm{p}$.

13. Maurin K. Methods of Hilbert spaces. Warsaw: PWN, 1967, 553 p. ISBN: 9780900318061 . Translated to Russian under the title Metody gil'bertova prostranstva. Moscow: Mir Publ., 1965, $570 \mathrm{p}$.

14. Ladyzhenskaya O.A. The boundary value problems of mathematical physics. N Y: Springer-Verlag, 1985, 332 p. ISBN: 978-1-4757-4317-3 . Original Russian text published in Ladyzhenskaya O.A. Kraevye zadachi matematicheskoi fiziki. Moscow: Nauka Publ., 173. 407 p.

15. Il'in A.M. Matching of asymptotic expansions of solutions of boundary value problems. Providence: American Mathematical Society, 1992, 281 p. ISBN: 978-0-8218-4561-5 . Original Russian text published in Il'in A.M. Soglasovanie asimtoticheskikh razlozhenii reshenii kraevykh zadach. Moscow: Nauka Publ., $1989,336 \mathrm{p}$.

16. Vishik M.I., Lyusternik L.A. A regular degeneration and boundary layer for linear differential equations with a small parameter. Uspekhi Mat. Nauk, 1957, vol. 12, no. 5, pp. 3-122 (in Russian).

17. Il'in A.M. A boundary layer. Itogi Nauki i Tekhniki. Ser. Sovrem. Probl. Mat. Fund. Napr., 1988, vol. 34, pp. 175-213 (in Russian).

Received December 14, 2019

Revised January 10, 2020

Accepted January 14, 2020

Aleksei Rufimovich Danilin, Dr. Phys.-Math. Sci., Prof., Krasovskii Institute of Mathematics and Mechanics of the Ural Branch of the Russian Academy of Sciences, Yekaterinburg, 620108 Russia, e-mail: dar@imm.uran.ru .

Cite this article as: A. R. Danilin. Asymptotics of a solution to a problem of optimal boundary control with two small cosubordinate parameters, Trudy Instituta Matematiki i Mekhaniki URO RAN, 2020, vol. 26, no. 1, pp. 102-111. 